\title{
Ultra-Low Voltage Analog IC Design: Challenges, Methods and Examples
}

\author{
Viera STOPJAKOVA, Matej RAKUS, Martin KOVAC, Daniel ARBET, \\ Lukas NAGY, Michal SOVCIK, Miroslav POTOCNY
}

\begin{abstract}
Department of IC Design and Test, Institute of Electronics and Photonics, Faculty of Electrical Engineering and Information Technology,

Slovak University of Technology, Ilkovičova 3, 81219 Bratislava, Slovakia

viera.stopjakova@stuba.sk
\end{abstract}

Submitted February 26, 2018 / Accepted February 27, 2018

\begin{abstract}
The paper brings an overview of main challenges and design techniques effectively applicable for ultra-low voltage analog integrated circuits in nanoscale technologies. New design challenges linked with a low value of the supply voltage and the process fluctuation in nanotechnologies, such as device models, robustness to process variation, device mismatch and others are discussed firstly. Then, design techniques and approaches to analog integrated circuits towards (ultra) low-voltage systems and applications are described. Finally, examples of basic building blocks of ultra-low voltage analog ICs designed in standard CMOS technology using such design techniques are presented. Finally, the developed circuits are compared to the state-of-the-art solutions in terms of the main parameters and features.
\end{abstract}

\section{Keywords}

Ultra-low supply voltage, analog design, integrated circuits, ultra-low voltage design techniques, bulk-driven

\section{Introduction and Motivation}

Recent enormous advance of semiconductor fabrication technology has allowed steep trends in the shrinkage rate of integrated circuits (IC) die area. According to [1], in 2017, there was fabricated a chip in $10 \mathrm{~nm}$ CMOS process node with the density of 100 millions of transistors per $1 \mathrm{~mm}^{2}$. Such an integration density brings great enhancement of computational power per area. However, this trend brings also crucial drawback, as downscaled process nodes suffer from the random fluctuation of process parameters, voltage and temperature sensitivity (PVT) along different samples on wafer.

Design of low-volatge (LV)/ultra-low voltage (ULV) and low-power (LP) analog and mixed-signal ICs in modern nanotechnologies represents a real challenge for circuit designers and researches, since it introduces several limitations in numerous aspects. Firstly, since advanced nanoscale technologies offer a possibility to design analog, digital, and radio-frequency (RF) circuits as well as micro-electromechanical systems (MEMS) on a single chip, there is usually issue of a common value of the supply voltage. With the technology development, the value of the supply voltage is scaled down significantly. However, the threshold voltage $\left(V_{\mathrm{TH}}\right)$ of MOS devices is not lowered with the same pace. This fact reduced the voltage headroom for conventional circuit topologies (e.g. cascode structures) to operate correctly. Moreover, low value of the supply voltage influences the main parameters of analog ICs, such as dynamic range (DR), power supply rejection (PSR), noise immunity, etc. Another limiting factor lies in the significant fluctuation of process parameters in nanoscale technologies, which brings a new requirement to IC design - circuits have to be robust enough against process, temperature and voltage variations.

Advanced nanoscale fabrication processes bring several limitations to ULV analog IC design. Firstly, it severely constrains the level of inversion the MOS transistors operate in. Resulting in higher mismatch between transistors, higher temperature sensitivity, lower transition frequency, much higher area requirements and many more [2]. The second issue lies in limited possible number of stacked transistors in order to ensure an operation in saturation region. According to [3], theoretical lower limit for saturation voltage of MOS transistor is defined as $V_{\mathrm{DSsat}(\min )} \approx 4 k T / q$, which at room temperature equals about $105 \mathrm{mV}$. Hence, the novel circuit topologies are heavily modified and their mode of operation is usually far from conventional approach. The third restriction is the dynamic voltage range, which affects the signal-to-noise ratio. This is usually compensated by railto-rail operation or differential signal representation for the price of circuit complexity.

In order to overcome these limitations, new ULV design techniques based on unconventional approaches have to be employed. Additionally, brand new topologies of basic building blocks for analog ICs, which offer reliable operation at ultra-low supply voltage conditions have to be developed. Towards improving the main parameters of ULV analog ICs, the fully differential signal processing is usually needed. 
The rest of the paper is organized as follows. In Sec. 2, new challenges in ULV analog IC desing in nanotechnologies are addressed. Section 3 brings an overview of design techniques and approaches to analog IC design that can be used to overcome serious limitations linked with a low value of the power supply and process fluctuations. Examples of ULV analog building blocks and their comparison to the state-ofthe-art achievements are presented within Sec. 4. Finally, some conclusions are given in Sec. 5 .

\section{Challenges in ULV Design}

As mentioned above, there are several different negative outcomes that are traded for low-power consumption or lowvoltage operation in age of battery-powered devices. In the following sections, the main challenges and issues of ULV analog IC design in nanotechnologies will be addressed in more details.

\subsection{Inversion Modes of a MOS Device}

MOS transistor can operate in different modes, depending on the level of inversion in the channel [4]. In strong inversion, the channel is fully formed, which is not the case in weak inversion. The transition between these two regions of operation is progressive, through a range called moderate inversion. The transistor behavior is rather different in the strong and weak inversion regions, which can be observed in the change from the square-law transfer characteristic in the former, to the exponential one in the latter. According to [5], the mode of MOS operation can be described using the inversion coefficient $i c$, calculated using (3), where $I_{\mathrm{f}}$ and $I_{\mathrm{r}}$ are the forward and reverse components of the drain current $I_{\mathrm{D}}$ respectively, as defined in (1). The specific current $I_{\text {spec }}$ is described in (2), where $n$ is the slope factor, $\mu$ is the mobility of current carriers, $C_{\mathrm{ox}}$ is the oxide capacitance per area, $W$ is the channel width, $L$ is the channel length and $U_{\mathrm{T}}$ is the thermal voltage. The transistor operates in strong inversion for $i c \gg 1$, in weak inversion for $i c \ll 1$, and in moderate inversion for $i c \cong 1$.

$$
\begin{gathered}
I_{\mathrm{D}}=I_{\mathrm{f}}-I_{\mathrm{r}}, \\
I_{\mathrm{spec}}=2 n \mu C_{\mathrm{ox}} \frac{W}{L} U_{\mathrm{T}}^{2}, \\
i c=\max \left(i_{f}=I_{\mathrm{f}} / I_{\mathrm{spec}}, i_{r}=I_{\mathrm{r}} / I_{\mathrm{spec}}\right) .
\end{gathered}
$$

In conventional analog IC, transistors operate in strong inversion [6]. However, in modern CMOS nanoscale technologies, the supply voltage has been significantly decreased to avoid high electric fields within the ever smaller devices and the related unwanted effects [5]. However, the threshold voltage was not scaled proportionally, as this would have resulted in increased leakage currents [7] and thus, larger power consumption [8]. In Fig. 1, the trend of the supply voltage $V_{\mathrm{DD}}$ and the threshold voltage $V_{\mathrm{TH}}$ reduction for shorter transistor channel lengths can be observed. It shows that while

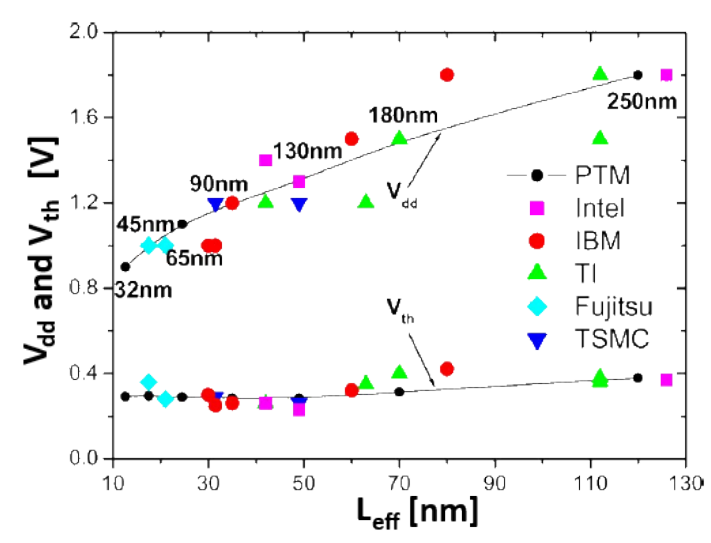

Fig. 1. Trend of supply $\left(V_{\mathrm{dd}}\right)$ and threshold $\left(V_{\mathrm{th}}\right)$ voltage scaling vs the effective transistor channel length $\left(L_{\mathrm{eff}}\right)[8]$.

$V_{\mathrm{DD}}$ has changed drastically, $V_{\mathrm{TH}}$ has remained almost constant. This leads to the reduction of the voltage headroom, which consequently limits the available overdrive voltage. This is especially important in circuits utilizing stacked transistors, such as various cascode structures commonly used in basic analog building blocks. The use of stacked transistors increases the minimum supply voltage required to achieve strong inversion in the circuits to many times more than $V_{\mathrm{TH}}$. As shown in Fig. 1, this condition can not be fulfilled in modern technologies. To utilize known topologies in these conditions, the operating point of the transistors is moved from strong to moderate or weak inversion.

An advantage of using transistors operating in weak inversion is the significantly reduced power consumption in comparison to those working in strong inversion. However, this decreases the bandwidth and increases the size of the transistors, as will be described later. Therefore, the use of transistors in weak inversion is favourable mainly for lowfrequency and low-power applications.

\subsection{Device Models}

Advanced nanoscale CMOS technologies brought a need for new design approaches and new simulation models of MOS devices that would be more accurate in the whole range of inversion regions. Therefore, to design circuits operating in these conditions, a new type of MOS transistor models is needed. A good example is the charge-based EKV model that accurately predicts the behaviour of MOS transistors in all inversion types [5], [9]. EKV model uses the smallest number of core parameters needed for a proper and accurate behavioural modelling. EKV model uses one equation for all inversion regions (including weak, moderate, and strong inversions). This model was successfully applied to low-voltage and low-power IC design with good accuracy. Moreover, it is very useful for analog circuit designer because significantly simplifies hand-made calculations. The basis of this model is parameter $g_{m} / I_{\mathrm{D}}$, which expresses the transconductance efficiency of a MOS transistor and describes how effectively the current (power) is transformed to the device transconductance. 


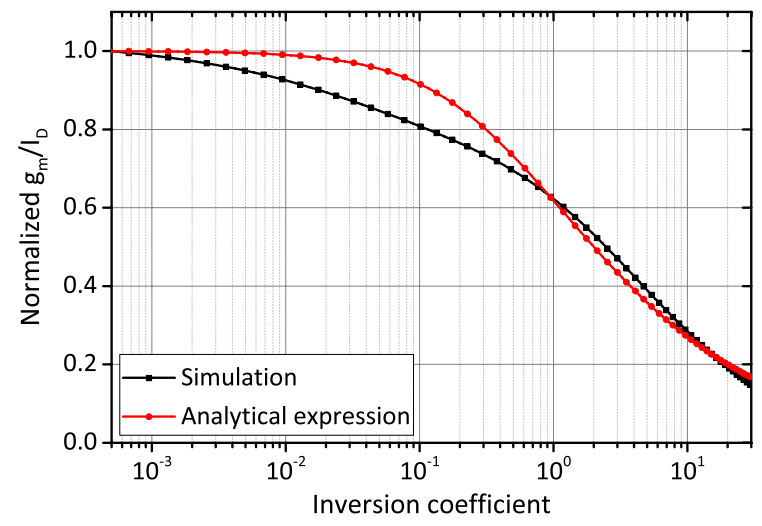

Fig. 2. Normalized $g_{m} / I_{\mathrm{D}}$ vs inversion coefficient $i c$.

There are several equations describing the relationship between the $g_{m} / I_{\mathrm{D}}$ parameter and the inversion coefficient $i c$ that defines the particular inversion region. These equations differ in interpolation accuracy of the transition region between the individual inversion regions. The simplest expression of $g_{m} / I_{\mathrm{D}}$ is given in [3]

$$
\frac{g_{m}}{I_{\mathrm{D}}}=\frac{1}{n U_{\mathrm{T}}} \frac{1}{0.5+\sqrt{0.25+i c}}
$$

where $n$ is the MOS transistor slope factor and $U_{\mathrm{T}}$ is the thermal voltage. Parameter $g_{m} / I_{\mathrm{D}}$ is not technology-related, which predicts its wide usability over different CMOS technologies. Nevertheless, simulation analysis of this characteristic using the widely used BSIM transistor models shows an inaccuracy in the weak inversion and moderate inversion regions. More details about $i c$ will be given in Sec. 3.4. Comparison of the characteristics obtained by analytical method and simulation shows significant error in these regions in a standard $130 \mathrm{~nm}$ CMOS technology, as can be observed in Fig. 2.

\subsection{Robustness to PVT Fluctuations}

With downscaling the process technology, it is rather difficult to ensure the designed geometric dimensions, doping profile or dielectric layer thickness of fabricated chips. These physical variations obviously reflect themselves on electrical parameters of ICs. The MOS transistor threshold voltage $V_{\mathrm{TH}}$ is one of the most essential electrical parameters of ICs, which is in this way degraded. The standard deviation of $V_{\mathrm{TH}}$ in $45 \mathrm{~nm}$ CMOS process node reaches approximately $16 \%$ of the mean value [10]. Other affected electrical parameters include parasitics of the chip interconnects, namely parasitic impedance and parasitic capacitance [11]. The characteristics of semiconductor structure alter with time, so electrical parameters of ICs are also affected by ageing. This creates a long-term drift, causing a systematic error. Such an effect is further described by the phenomenon of negative-bias temperature instability (NBTI) [12].

Recent ICs needs to meet industrial requirements, hence the circuit topologies need to be robust against temperature variation in a wide range, at least from $-20^{\circ} \mathrm{C}$ to $85^{\circ} \mathrm{C}$. Such

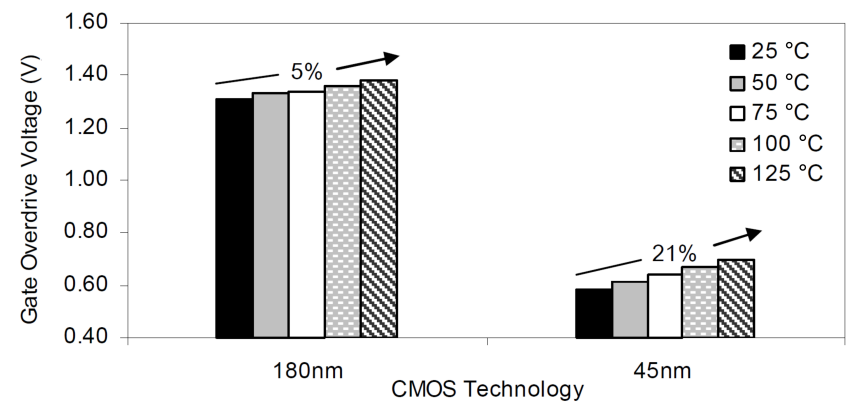

Fig. 3. The variation of the transistor overdrive voltage with temperature for two technology nodes [13].

variations cause substantial shift of the semiconductor structures electric characteristics. Thus, it obviously results in another deviation and systematic error in IC electric parameters.

Advanced integrated systems impose challenging demands on IC power consumption. Since the threshold voltage does not scale consistently with the supply voltage, the $V_{\mathrm{DD}} / V_{\mathrm{TH}}$ ratio has decreased from approximately 3 for $800 \mathrm{~nm}$ technology node to level of 2 for $45 \mathrm{~nm}$ technology node [13]. Moreover, the voltage headroom problem get worse with temperature. As can be observed from Fig. 3, the temperature sensitivity of the transistor overdrive voltage in $45 \mathrm{~nm}$ technology is higher than the one in $180 \mathrm{~nm}$ node [13].

Additionally, similar tendency has been presented in terms of the MOS transistor drain current $\left(I_{\mathrm{D}}\right)$ in [14]. It was shown that the $I_{\mathrm{D}}$ temperature sensitivity increases in the inverse proportion with $V_{\mathrm{DD}}$. Also, the $I_{\mathrm{D}}$ sensitivity to $V_{\mathrm{DD}}$ increases dramatically below the supply voltage value of approx. $500 \mathrm{mV}$. However, this change is less significant with technology downscale as the temperature sensitivity.

\subsection{Supply Voltage Scaling}

Contemporary trends in CMOS technologies include the device downscaling and shrinking the gate-oxide thickness down to the order of few nanometers. This leads to low breakdown voltages, and therefore, the supply voltage has to be reduced to ensure the circuit correct operation and the reliability. Reducing the device size and technology resolution also leads to increased density of components on chip. Since the substrate can dissipate only certain amount of heat per area, the power consumption has to be reduced too. On the other hand, the decrease of the MOS transistor threshold voltage is less significant in comparison to the supply voltage scaling over the years, which results in worse on/off characteristics and lower voltage swing.

The minimum $V_{\mathrm{DD}}$ of a CMOS circuit is limited by the value given by a sum of transistor turn-on voltage $V_{\mathrm{GS}}$ and the required voltage swing. For an NMOS device in a standard $130 \mathrm{~nm}$ CMOS process, $V_{\mathrm{TH}} \approx 300 \mathrm{mV}$ is quite typical value to achieve saturation in the strong inversion region. Subsequently, when two or more NMOS transistors are stacked into cascode, there is low or no headroom left if using low supply voltages $\left(V_{\mathrm{DD}} \leq 1 \mathrm{~V}\right)$. 
Multi-threshold process or BiCMOS technology can be used to achieve better performance but at the cost of higher fabrication expenses. However, advanced techniques for IC design in a standard CMOS process have been developed to compete these technologies. Nevertheless, IC design becomes rather difficult when it comes to low-voltage and lowpower operation. On the other side, to achieve comparable gain of simple stages in comparison to cascode structures, it is possible to connect multiple simple stages in series (into cascade). However, multi-gain stage topologies may represent a stability problem due to the location of their dominant poles. Subsequently, clever compensation techniques has to be used to ensure stability of circuits.

Another problem in ULV IC design is degradation of the dynamic range. Dynamic range is ratio between the maximum voltage swing (or supply voltage) and noise floor. Since noise is relatively constant, lowering the power supply voltage leads to the dynamic range degradation.

\subsection{Mismatching}

The operation of high performance ICs strongly depends on the circuit element layout matching and differential paths matching. Therefore, any random or systematic deviations invoked by effects described above actually cause mismatch, which consequently decreases the production yield or/and the IC reliability. As an example of undesired circuit parameters caused by mismatching, the input offset voltage of an operational amplifier can be given. This parameter is of the significant concern in the amplifier design, as it is tightly related to degradation of other AC or DC amplifier specifications. The situation get even worse for low-voltage conditions. Other important parameters that are degraded due to both the mismatch in nanotechnologies and low-power restrictions, include common-mode rejection ratio and power supply rejection ratio. For this reason, appropriate layout techniques have to be employed in order to reduce the mismatch of all devices working with a low power supply value.

\section{ULV Design Techniques}

Generally, very common and useful approach in ULV IC design is using the differential or balanced structures thanks to their high values of PSRR and CMRR, higher voltage swing and lower distortion.

There have been several design techniques developed for ULV and low-power analog ICs. The most used ones include:

- Floating-gate MOS transistors

- Self-cascode topologies

- Level shifters

- MOS transistors operating in sub-threshold region

- Bulk-driven MOS transistors

- Dynamic-threshold MOS transistors

\subsection{Floating-gate MOS Transistor}

Floating-gate MOS (FGMOS) transistor is similar to the conventional MOS transistor. The difference is in the isolated floating-gate (FG) with no resistive connections. Secondary gates (inputs) are then deposited above, which are connected to FG only through capacitive coupling, since the FG is completely surrounding with high resistive material (usually $\mathrm{SiO}_{2}$ ) [15].

Floating-gate voltage $V_{\mathrm{FG}}$ is not controlled directly but through the input gates with capacitive coupling. $V_{\mathrm{FG}}$ can be expressed as

$$
\begin{aligned}
V_{\mathrm{FG}}=\frac{Q_{\mathrm{FG}}+C_{\mathrm{FG}, \mathrm{D}} V_{D}+C_{\mathrm{FG}, \mathrm{S}} V_{S}+}{C_{\Sigma}} & \\
+\frac{\sum_{\mathrm{FG}, \mathrm{B}} V_{\mathrm{B}}}{C_{\mathrm{Gi}} V_{\mathrm{Gi}}} &
\end{aligned}
$$

where $Q_{\mathrm{FG}}$ is the static charge on the floating-gate and $C_{\sum}$ represents the total capacitance seen at the floating-gate, which is given by 6

$$
C_{\Sigma}=C_{\mathrm{FG}, \mathrm{D}}+C_{\mathrm{FG}, \mathrm{S}}+C_{\mathrm{FG}, \mathrm{B}}+\sum_{i=1}^{n} C_{\mathrm{Gi}} .
$$

All these capacitances can be observed in the equivalent circuit shown in Fig. 4.

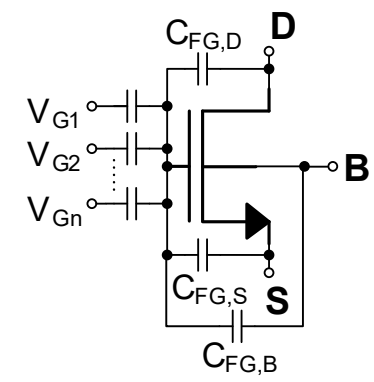

Fig. 4. Input and parasitic capacities of a FGMOS transistor.

Since the electrical isolation between the input gates and floating-gate is almost ideal, the static charge $Q_{\mathrm{FG}}$ can remain on the FG for years with negligible variation from the original value. The equivalent threshold voltage of a FGMOS transistor can be controlled by several ways [15]: 1) Ultra-violet light, which can cause the temporary conductivity of the isolation layer, 2) Injection of hot electrons (it requires rather big programming currents), 3) Fowler-Nordheim tunnelling (it requires relatively high programming voltages).

\subsection{Self-cascode Structures}

In ULV conditions, regular cascode structures are avoided as their use decreases the output signal swing. The self-cascode (Fig. 5) is a two-transistor structure, which can be treated as a single transistor. Gates of both transistors are driven by a common input signal [16]. The self-cascode technique offers higher output impedance and reduces the effect of Miller capacitance on the transistor gates. Moreover, this structure creates higher voltage headroom for signal processing. 


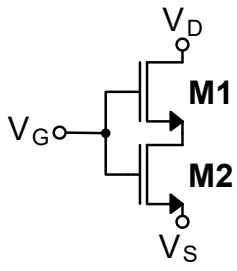

Fig. 5. The self-cascode structure.

The lower (upper) transistor M2 (M1) operates out of saturation (in saturation). If both transistors have similar aspect ratio $(W / L), \mathrm{M} 2$ will operate in the linear region and M1 will operate in saturation. In such a case, the selfcascode works like a common-source stage with higher voltage gain. On the other hand, for $(W / L)_{1}>>(W / L)_{2}$, such a circuit behaves like a single MOS transistor operating in the saturation region with rather high gain and significantly lower influence of the channel length modulation. The output resistance $r_{\text {out }}$ of this topology is approximately proportional to $(W / L)_{1} /(W / L)_{2}$ ratio, and the saturation voltage $V_{\mathrm{DS} \text { (sat) }}=V_{\mathrm{GS}}-V_{\mathrm{TH}}$ is roughly comparable to the conventional MOS transistor. The basic principle of this technique is in different threshold voltages of the transistors (i.e. $V_{\mathrm{TH} 1} \neq V_{\mathrm{TH} 2}$ ), which is not available in a standard low-cost CMOS process [16].

\subsection{Voltage Level Shifters}

Dynamic level shifters represent also a potential solution for circuits with low-voltage input signals. This technique usually uses resistors for shifting the input commonmode voltage to the operation region of input differential transistor pair. Thus, the input transistor pair keeps sufficient transconductance $g_{m}$ in comparison to the other low-voltage design techniques [17]. Other approach uses a single-supply level shifters for LP applications. Such shifters do not suffer from delay variation (due to different current driving capabilities of transistors), high power consumption and failures at low supply voltage $V_{\mathrm{DDL}}$, compared to the conventional dual-supply equivalents. Single-supply level shifters have advantages over the dual-supply ones in terms of pin count, congestion in routing and the overall cost of the system. Another benefit of the single-supply lever shifters is flexible placement and routing in physical design [18].

\subsection{MOS Transistors in Sub-threshold Region}

Operating region of the MOS transistor becomes one of the most important aspects for ULV analog IC design [19]. The inversion region of the MOS device is defined by the inversion coefficient $i c$ expressed by

$$
i c=\frac{I_{\mathrm{D}}}{I_{0}(W / L)}=\frac{I_{\mathrm{D}}}{2 \mu C_{\mathrm{ox}} U_{\mathrm{T}}^{2}(W / L)}
$$

where $I_{\mathrm{D}}$ is the transistor drain current, $I_{0}$ is the technological current, $\mu$ is the charge-carrier mobility, $C_{\mathrm{ox}}$ is capacitance of the gate-oxide and $U_{\mathrm{T}}$ is the thermal voltage.

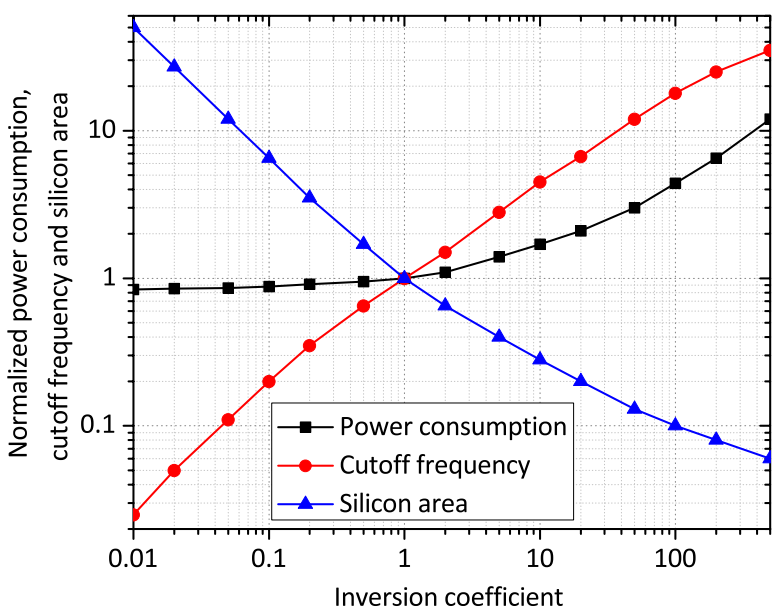

Fig. 6. Normalized power consumption, cut-off frequency and area vs. the inversion coefficient [20].

MOS transistor operating in strong (weak) inversion region has good (bad) frequency response, small (large) chip area and high (low) power consumption. Extreme cases of the strong or weak inversion regions do not provide good trade-off between these variables. The solution could be to use the moderate inversion region. MOS transistor operates in strong (weak) inversion for $i c>10$ (ic $<0.1)$. Everything in between belongs to the moderate inversion with center in $i c=1$ (when $\left.I_{\mathrm{D}}=I_{0}\right)$, in logarithmic scale. Figure 6 shows the relation between the normalized speed, the power consumption and chip area [20].

$g_{m} / I_{\mathbf{D}}$ approach - A conventional MOS transistor simulation models use different equations for the weak and strong inversion regions. The transition moderate inversion is often described using difficult equation, and it is impossible to avoid significant inaccuracies and discontinuities. Therefore, a new design approach using parameter $g_{m} / I_{\mathrm{D}}$ is increasingly used [21]. The advantage of this approach is that MOS transistor sizing rules for IC design are defined easily. Such approach can be used for any inversion region thanks to the "one-equation" transistor model. Relation between $g_{m} / I_{\mathrm{D}}$ and the inversion coefficient $i c$ (Fig. 4) became fundamental for analog IC design in submicron and nanoscale technologies. Parameter $g_{m} / I_{\mathrm{D}}$ suggests the operation region of a MOS transistor and, more importantly, $g_{m} / I_{\mathrm{D}}$ does not change across different technologies.

\subsection{Bulk-driven MOS Transistors}

Drain current $I_{\mathrm{D}}$ of a conventionally connected MOS transistor is usually controlled by the gate-source voltage $V_{\mathrm{GS}}$. In the bulk-driven (BD) design technique, the bulk electrode of the MOS transistor is used as a signal input to modulate drain current (Fig. 7) [22].

Using bulk-source voltage $V_{\mathrm{BS}}$ instead of $V_{\mathrm{GS}}$ may introduce unwanted body transconductance $g_{m b}$ [23], which is 3-4 times lower than the gate transconductance $g_{m}$. The body transconductance $g_{m b}$ is a small-signal parameter of the MOS transistor and it can be expressed as 


\begin{tabular}{|l|c|c|c|c|}
\hline Technique & GBW & Supply voltage & Power consumption & Technology \\
\hline FGMOS & Medium & $<V T N+|V T P|$ & High & 2x Polysilicon \\
\hline Self-cascode & Medium & $<V T N+|V T P|$ & Medium & Multi-threshold \\
\hline Bulk-driven & Low & $<V T N+|V T P|$ & Low & Standard (triple-well) \\
\hline Dynamic-threshold & Medium & $<V T N+|V T P|$ & Low & Standard (triple-well) \\
\hline Sub-threshold & Low & $<V T N+|V T P|$ & Low & Standard \\
\hline Level shifters & High & $<V T N+|V T P|$ & Medium & Standard \\
\hline
\end{tabular}

Tab. 1. Comparison of LV design techniques in terms of main parameters.

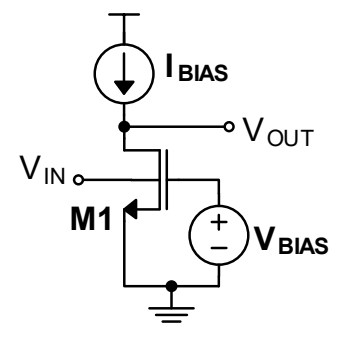

Fig. 7. Schematic of a bulk-driven MOS transistor.

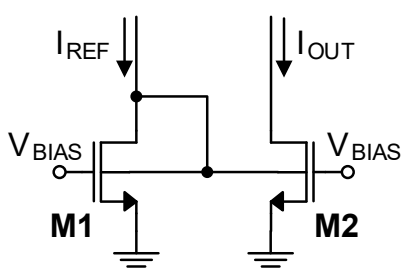

Fig. 8. Simple bulk-driven current mirror.

$$
g_{m b}=\frac{\partial i_{\mathrm{d}}}{\partial v_{\mathrm{BS}}}=\frac{\gamma g_{m}}{2 \sqrt{-2 \phi_{\mathrm{F}}-V_{\mathrm{BS}}}}
$$

where $\phi_{\mathrm{F}}$ is the Fermi potential and $\gamma$ is the substrate coefficient. Using $g_{m b}$ instead of $g_{m}$ also leads to lower gain band-width and worse frequency response. Bulk-driven approach increases the input capacitance and input noise. There is also susceptibility to turning on an unwanted latch-up effect in this approach. Nevertheless, clever physical layout by following the specific layout rules can prevent the circuit against latch-up.

On the other side, the BD technique leads to a significantly reduced need to overcome the threshold voltage at the MOS transistor input. This increases the voltage headroom and makes the technique very useful for ULV analog IC design. Moreover, the main advantage of this technique is the compatibility with a standard CMOS process, hence there is no need to change the structure of the conventional MOS transistor [20]. BD design technique can be easily applied to different analog IC building blocks, such as current mirrors [24] or differential pairs [25]. For example, a simple current mirror designed using BD approach is shown in Fig. 8, where bulks of transistors M1 and M2 in both branches are tight together and gates are connected to the bias voltage $V_{\mathrm{BIAS}}$. The input current brought into the input branch creates the voltage $V_{\mathrm{BS}}$ between bulks and sources of both transistors.

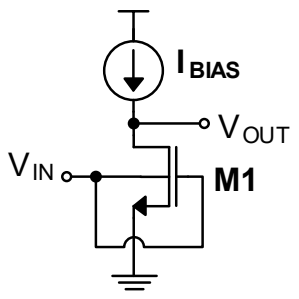

(a)

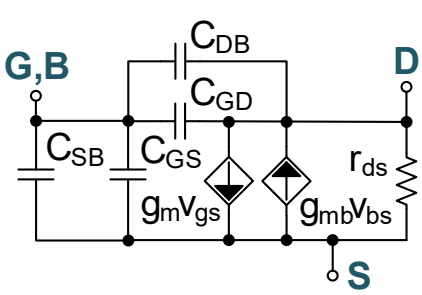

(b)
Fig. 9. DTMOS transistor: (a) schematics; (b) equivalent circuit.

\subsection{Dynamic-threshold MOS Transistors}

Dynamic-threshold (DT) MOS transistors are derived from the BD technique with only a simple difference in the gate biasing conditions. DT transistor has gate and bulk electrodes tight together and biasing is realized dynamically with the input signal swing, which leads to a dynamically reduced value of the threshold voltage $V_{\mathrm{TH}}$. Thanks to the dynamic biasing conditions, the potential of the conductive channel is controlled by both the gate and bulk at the same time, which results in a high overall transconductance $g_{m}+g_{m b}$ and faster current transfer. Schematic and small-signal equivalent circuit of the DT MOS transistor is depicted in Fig. 9(a) and Fig. 9(b), respectively [26].

Similarly to the BD technique, by applying the DT design technique to basic analog IC building blocks and widely used topologies, DT equivalents can be obtained.

\subsection{Summary of ULV Design Techniques}

Generally, the LV (ULV) design techniques described above have certain performance limitations such as gain bandwidth (GBW) degradation, increased input noise, increased input impedance or decreased overall transconductance. Main characteristics of the analyzed design techniques are summarized and compared in Tab. 1 [27].

Taking into account the feasibility of particular LV design techniques in different technologies, it is advantageous to use techniques employing MOS transistors operating in the sub-threshold region, such as BD MOS transistors and DT MOS transistors, because there is no need for modification of a standard CMOS process. Additionally, with those two LV techniques, also low power consumption of designed circuits can be achieved. 


\subsection{Calibration Techniques}

In order to overcome nanotechnology drawbacks and compensate the effects of PVT fluctuations on ULV circuits, calibration techniques are employed in analog IC design. Calibration methods are still developing, as new issues appear along with upcoming technology advances. Such methods can be differentiated as static and dynamic ones. Static calibration methods are represented mainly by trimming, where laser or current blown fuses are used [28]. Dynamic calibration methods include, for example, the chopper stabilization [29], auto-zeroing [30] or digital calibration [31]. The latter method will be more deeply described in Session 4.3.1, where digital calibration of a variable gain amplifier was implemented, with a focus on the input offset voltage compensation.

\section{Examples of ULV Circuits}

\subsection{Current Mirrors}

Current mirrors (CM) represent one of the most used basic building blocks of analog ICs. Therefore, in our analysis of low-voltage circuit examples, three basic topologies of current mirrors designed using the BD technique are compared to their standard gate-driven equivalents in terms of the output characteristics. Dimensions of transistors were $L=2 \mu \mathrm{m}$ and $W=6 \mu \mathrm{m}$ for a simple CM (Fig. 8), and $W=14 \mu \mathrm{m}$ for the improved Wilson and cascode current mirrors (Fig. 10). Gates of all BD transistors are biased by voltage $V_{\mathrm{BIAS}}=300 \mathrm{mV}$. The designed CMs were manufactured in a standard $130 \mathrm{~nm}$ CMOS technology and then, the measured parameters were compared to simulation results.

Since the minimum output voltage $V_{\mathrm{MIN}}=V_{\mathrm{DSs}}$ of the simple CM does not depend on the threshold voltage $V_{\mathrm{TH}}$ nor the transconductance, the output resistance $r_{\mathrm{out}}=r_{\mathrm{ds}}$ of both GD and BD simple current mirrors are similar. In other words, a convential gate-driven simple CM is suitable also for use in low-voltage ICS but its main drawback is rather low output resistance (in order of hundreds $\mathrm{k} \Omega$ ). Moreover, even lower output resistance (for similar value of $V_{\text {MIN }}$ ) was reported for measured results if compared to the simulation results Fig. 11.

The improved Wilson CM can enhance the output resistance of the mirror by adding a negative serial feedback to the circuit. From Fig. 12, one can observe that the BD technique reduces the $V_{\mathrm{MIN}}$ voltage from $300 \mathrm{mV}$ to approximately $120 \mathrm{mV}$, which is similar to the simple CM but with much higher output resistance (in order of $\mathrm{M} \Omega$ ).

In the case of cascode CM (Fig. 13), the minimum output voltage is again significantly reduced to the value about $150 \mathrm{mV}$ for the BD design topology. Measurements of both topologies proved simulated results with only slight inaccuracy observed. Thus, DC biasing conditions had to be slightly modified from $300 \mathrm{mV}$ to $320 \mathrm{mV}$ to achieve the required accuracy of the cascode mirror. More details on the LV current mirror analysis can be found in [32].

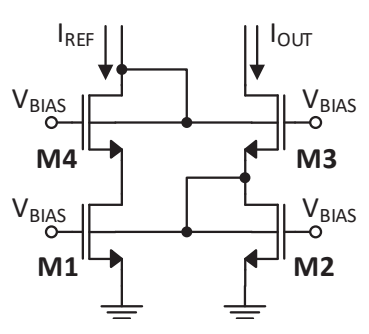

(a)

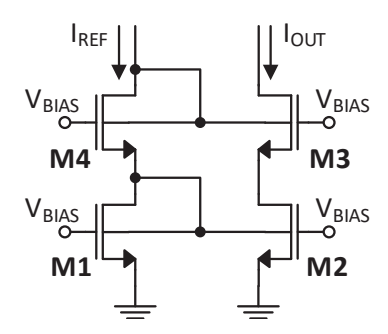

(b)
Fig. 10. Schematics of the bulk-driven CMs: (a) improved Wilson; (b) cascode.

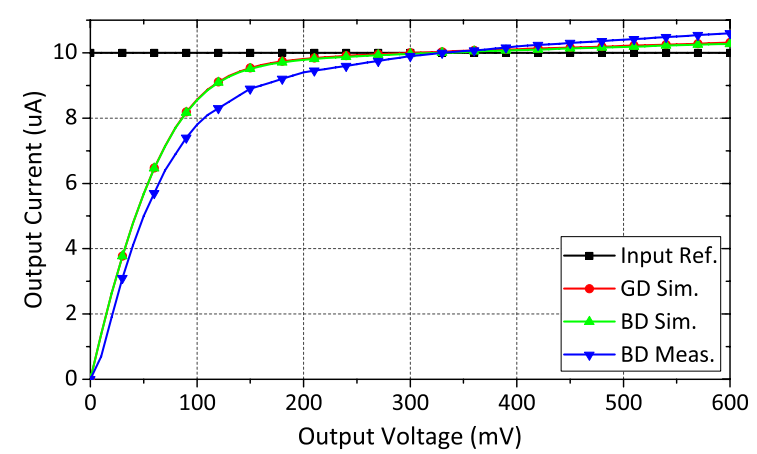

Fig. 11. Output characteristics of a simple CM (using GD and BD design styles).

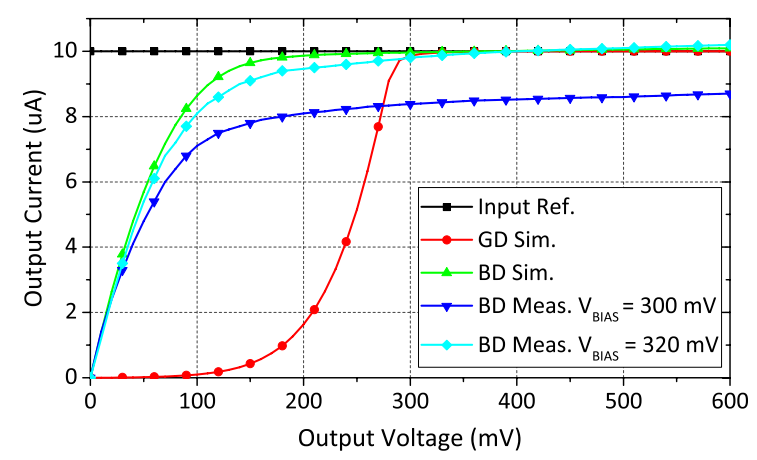

Fig. 12. Comparison of the improved Wilson CMs results.

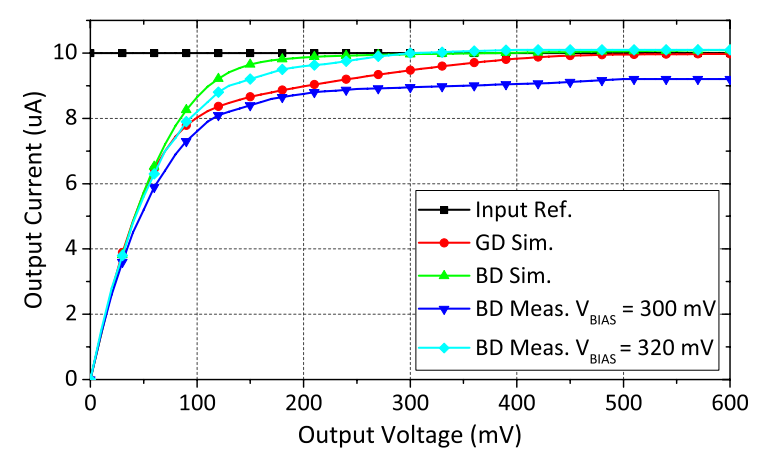

Fig. 13. Output characteristics of the cascode CM.

Presented experimental results have proven that the BD design approach can increase the signal swing by reducing the MOS transistor dependence on the threshold voltage. This can be the key towards LV current mirror design, especially when the cascode CM topologies are used (due to their simplicity and favourable characteristics). 


\begin{tabular}{|l|c|c|c|c|c|c|c|}
\hline Parameter & [39] & [33] & [34] & [35] & [36] & [37] & [38] \\
\hline Process & $130 \mathrm{~nm}$ & $65 \mathrm{~nm}$ & $90 \mathrm{~nm}$ & $180 \mathrm{~nm}$ & $500 \mathrm{~nm}$ & $180 \mathrm{~nm}$ & $130 \mathrm{~nm}$ \\
\hline$V_{\mathrm{DD}}$ & $0.4 \mathrm{~V}$ & $1-1.2 \mathrm{~V}$ & $1 \mathrm{~V}$ & $1.8 \mathrm{~V}$ & $1.8-5 \mathrm{~V}$ & $0.3 \mathrm{~V}$ & $0.2 \mathrm{~V}$ \\
\hline Design style & $\mathrm{BD}$ & $\mathrm{GD}$ & $\mathrm{GD}$ & GD current inp. & GD & BD & GD \\
\hline Total power & $94 \mathrm{nW}$ & $60 \mathrm{fJ} / \mathrm{decision}$ & $40 \mu \mathrm{W}$ & $1.01 \mathrm{~mW}$ & $40 \mathrm{nA}$ & $100 \mathrm{pW}$ & $3 \mathrm{fF} / \mathrm{decision}$ \\
\hline Frequency & $500 \mathrm{kHz}$ & $1 \mathrm{MHz}$ & $1 \mathrm{GHz}$ & $500 \mathrm{MHz}$ & $10 \mathrm{kHz}$ & $62.5 \mathrm{kHz}$ & $250 \mathrm{kHz}$ \\
\hline Load & $25 \mathrm{pF}$ & - & - & $200 \mathrm{fF}$ & - & - & $10 \mathrm{fF}$ \\
\hline Silicon area & $410 \mu \mathrm{m}^{2}$ & $100 \mu \mathrm{m}^{2}$ & $543.75 \mu \mathrm{m}^{2}$ & - & - & - & - \\
\hline Sim./Meas. & Meas. $\left(25^{\circ} \mathrm{C}\right)$ & Meas. $\left(25^{\circ} \mathrm{C}\right)$ & Meas. $\left(25^{\circ} \mathrm{C}\right)$ & Sim. corners & Meas. $\left(25^{\circ} \mathrm{C}\right)$ & Sim. $\left(25^{\circ} \mathrm{C}\right)$ & Sim. $\left(25^{\circ} \mathrm{C}\right)$ \\
\hline
\end{tabular}

Tab. 2. Comparison of state-of-the-art ULV comparators.

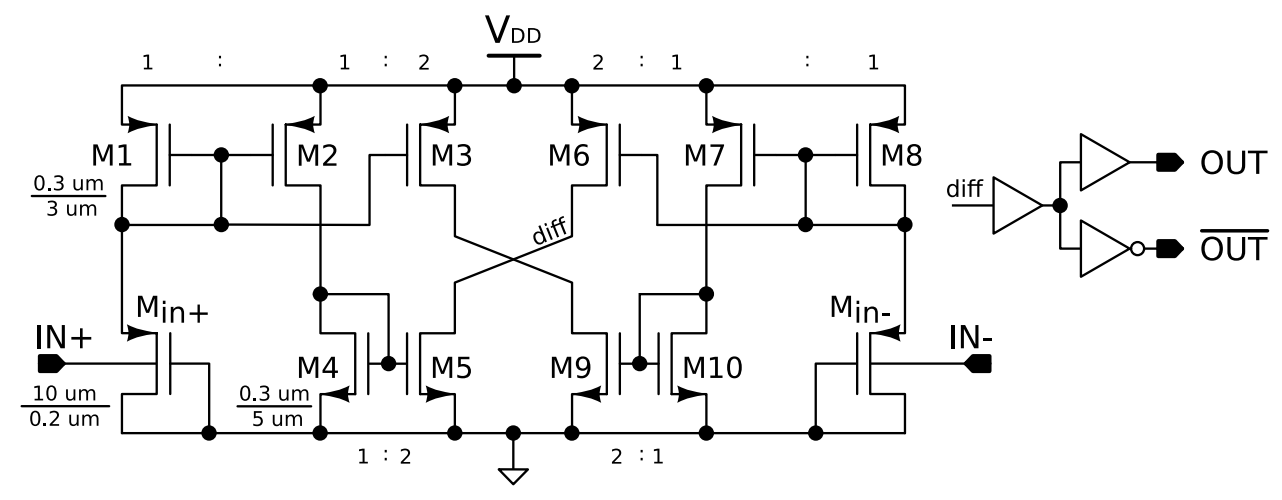

Fig. 14. A non-latched LV rail-to-rail bulk-driven voltage comparator in $130 \mathrm{~nm}$ CMOS technology [39].

\subsection{Low-voltage Comparators}

Several low-voltage comparator designs with the low power consumption have been developed mainly in deep submicron CMOS technologies thanks to fair trade-off between the cost, fabrication process properties, and component parameters. The research and the comparison of state-of-the-art (ultra) low-voltage and low-power comparator designs was carried out. The results of the investigation are summarized in Tab. 2.

A novel low-voltage comparator design that we have proposed in [39] is depicted in Fig.14 (along with the device dimensions). Standard $130 \mathrm{~nm}$ CMOS process was selected and the power supply voltage of $400 \mathrm{mV}$ was used. The input stage is comprised of bulk-driven PMOS transistors, which introduces the rail-to-rail input voltage range ability. Again, the real limitation of true rail-to-rail range is the offset voltage. The input voltage modulates the current flowing through the input circuit branch. This current is afterwards mirrored by transistors M1-M5. Set mirroring ratios improve the consumed quiescent current and layout matching of respective devices. The differential voltage is generated in the node called diff. Since the analog part is symmetrical, the other half of the circuit works in the identical way. The differential voltage is shaped by serial system of buffers and inverters. The driving capability was set to $f=500 \mathrm{kHz}$ at capacitive load of $25 \mathrm{pF}$. The expected performance and other parameters are discussed in more details in [39].
The measurements performed on the fabricated prototype chip of the comparator (Fig. 14) confirmed correct operation and ability to work with even lower supply voltage value. Measured DC transfer characteristics at room temperature for various reference voltages is depicted in Fig. 15. The experimental measurement data are also compared to simulation results.

One can observe an excellent agreement between both sets of data. The calculated average input offset voltage equals $V_{\mathrm{OS}}=2.29 \mathrm{mV}$ while the average power consumption

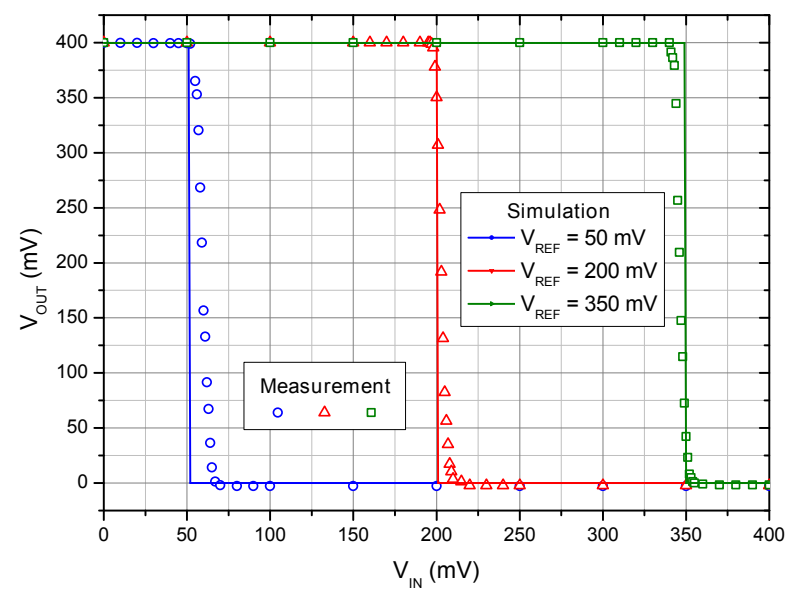

Fig. 15. Comparison of measured and simulated transfer characteristics of the LV comparator proposed in [39]. 


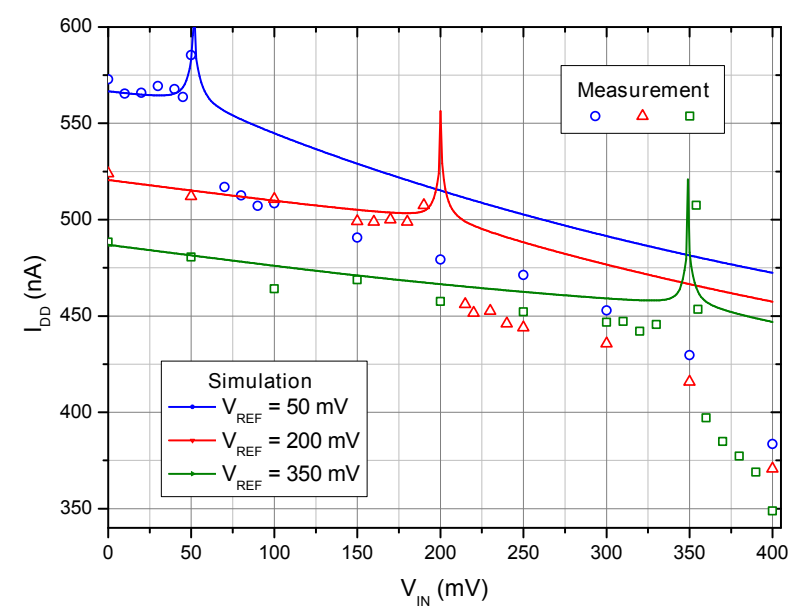

Fig. 16. Comparison of measured and simulated current consumption of the LV comparator proposed in [39].

was calculated at $P=94 \mathrm{nW}$. Figure 16 depicts measured current consumption of the proposed comparator along with the simulation results as well. There is an ongoing performance analysis of the proposed rail-to-rail bulk-driven comparator which should be published soon.

\subsection{Variable-gain Amplifier}

Variable-gain amplifier (VGA) represents a basic building block of many mixed-signal integrated systems. Novel ultra-low voltage VGA circuits, based on the BD approach, were presented in [25], [40], [41]. The general block diagram of the VGA presented in [25], [40] is depicted in Fig. 17. The two-stage VGA circuit is based on a BD differential difference amplifier (DDA). The second stage is composed of a standard common-source amplifier (CSA). Additionally, two common-mode feedback (CMFB) circuits were proposed and used in order to ensure a stable operational point of the first stage as well as the second stage. The developed VGA can reliably work at the supply voltage of $0.6 \mathrm{~V}$. The achieved tuning range is from $20 \mathrm{~dB}$ to $47 \mathrm{~dB}$, and the power consumption of about $11 \mu \mathrm{W}$ was reported. The developed VGA was successfully employed in an automatic gain control (AGC) loop.

Another experimental VGA circuit, designed to work with the supply voltage of $0.4 \mathrm{~V}$ with the power consumption of $3.4 \mu \mathrm{W}$, was presented in [41], and its block diagram is shown in Fig. 18. This VGA design is based on a pseudo differential difference amplifier (PDDA), which was designed using the BD technique.

In this ULV amplifier design, a common-mode feedforward (CMFF) circuits was used in order to ensure the bias voltage for the input BD transistors [41]. Besides, a CMFB circuit for the stabilization of the operational point was employed here as well. Since in the PDDA topology, the tail current source is missing, the CMFF circuit is used to improve the common-ode rejection ratio (CMRR) of the VGA. The tuning range of the amplifier presented in [41] is from $0 \mathrm{~dB}$ to $18 \mathrm{~dB}$ for the control voltage value from $0 \mathrm{~V}$ to $0.33 \mathrm{~V}$, respectively.

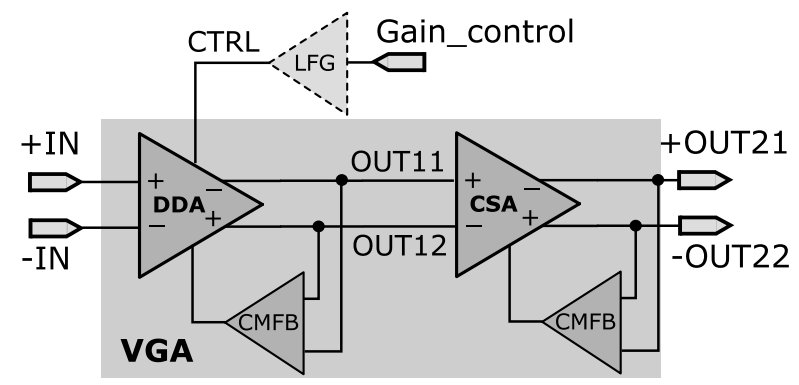

Fig. 17. General block diagram of the VGA presented in [40], [25].

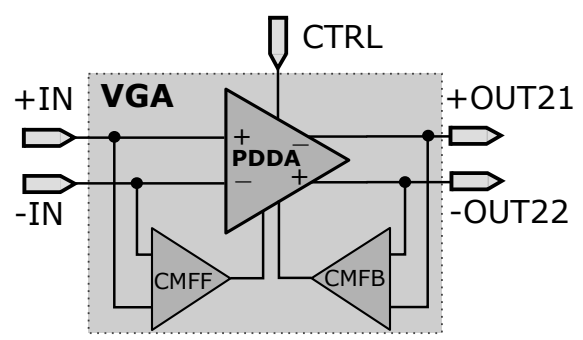

Fig. 18. Block diagram of the VGA presented in [41].

The main parameters of the ultra-low voltage VGAs designed using the BD technique are summarized and compared in Tab. 3.

\begin{tabular}{l|c|c|c} 
Parameter & {$[\mathbf{4 2}]$} & {$[\mathbf{2 5 , 4 0 ]}$} & {$[\mathbf{4 1}]$} \\
\hline Process & $180 \mathrm{~nm}$ & $130 \mathrm{~nm}$ & $130 \mathrm{~nm}$ \\
Design style & $\mathrm{BD}$ & $\mathrm{BD}$ & $\mathrm{BD}$ \\
Total power & $0.2 \mathrm{~mW}$ & $0.12 \mu \mathrm{W}$ & $3.45 \mu \mathrm{W}$ \\
$V_{\mathrm{DD}}$ & $0.8 \mathrm{~V}$ & $0.6 \mathrm{~V}$ & $0.4 \mathrm{~V}$ \\
Tuning & $17(\mathrm{~L}-\mathrm{in}-\mathrm{dB})$ & $14(\mathrm{~L}-\mathrm{in}-\mathrm{dB})$ & $8(\mathrm{~L}-\mathrm{in}-\mathrm{dB})$ \\
range [dB] & $-10.5 \div 6.5$ & $20 \div 47$ & $0 \div 18$ \\
Gain [dB] & $42 \div 195$ & $0.28 \div 0.29$ & 0.73 \\
BW [MHz] & THD: & THD $<$ & \\
& $0.32 \% @ \mathrm{in}:$ & $-30 \mathrm{~dB} @ 1 \mathrm{mV}$ & N/A \\
Distortion & $-16.2 \mathrm{dBV}$, & & Sim. (PV)
\end{tabular}

Tab. 3. Main parameters of the presented VGA designs.

\subsubsection{Digital Calibration of the VGA}

A digital calibration technique, targeting the compensation of the VGA input voltage offset $\left(V_{\mathrm{IN}-\mathrm{OFF}}\right)$, was employed and implemented within an experimental chip. The block diagram of the calibration system is depicted in Fig. 19. The whole system can be divided in three main functional parts: the calibrated device, compensation circuitry and the control logic. The VGA circuit was used as the calibrated device.

As already mentioned above, the VGA is a low-voltage fully differential difference amplifier. Its gain can be varied up to $47 \mathrm{~dB}$ and the GBW of $15.5 \mathrm{MHz}$ is reached. Thus, it is crucial to optimally adjust compensation circuitry for a specific range of $V_{\text {IN-OFF }}$ range. The residual offset of the proposed calibration method is directly proportional to the maximum offset value which this method is able to compensate. Specifically, this is represented by the DAC (digital-to- 


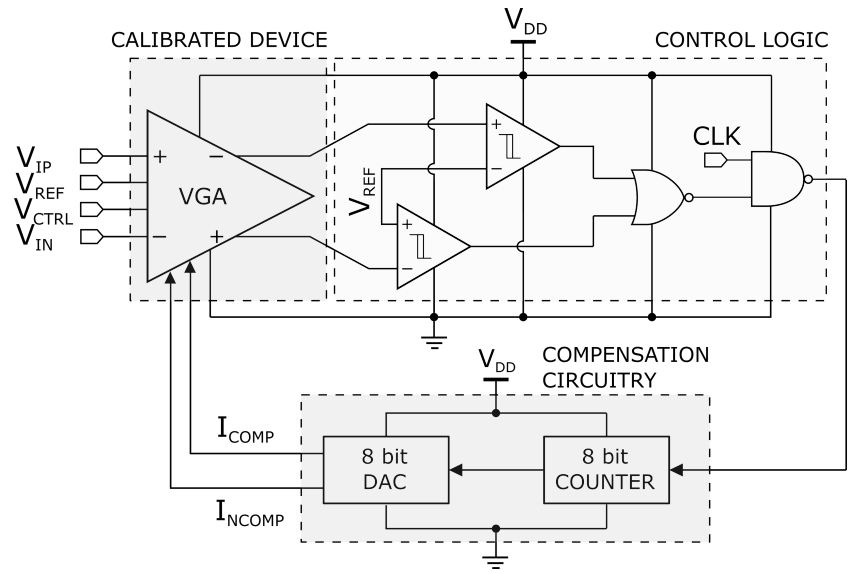

Fig. 19. The block diagram of the calibration system for VGA.

analog converter) specifications, analog response to a lowest significant bit $\left(a_{\mathrm{LSB}}\right)$, and the full scale. Therefore, it was essential to determine the statistical distribution of $V_{\text {IN-OFF }}$ over significant number of samples. Fig. 20 presents variation of

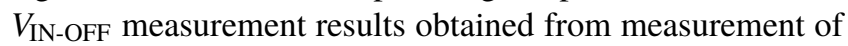
naked prototyped chips. The statistical distribution consisting of 60 samples reaches the mean value of $2.98 \mathrm{mV}$ and the standard deviation of $6.85 \mathrm{mV}$. The DAC full scale was adjusted to the $V_{\mathrm{IN}-\mathrm{OFF}}$ results in Fig. 20. Considering the $3 \sigma$ range of distribution, the calibration circuit was optimized to compensate the $V_{\mathrm{IN}-\mathrm{OFF}}$ of $\pm 20 \mathrm{mV}$.

The main part of compensation circuitry is a 8-bit DAC with the differential output, which is connected to the VGA so that it can modify the current-voltage conditions in both branches of the amplifier. The DACs input code is set by an 8-bit counter. Initially, the input code is 0 and corresponding differential outputs of the DAC are 0 (the minimum output current) and the full scale (the maximum output current). The output offset voltage of the VGA (VOUT-OFF) is, in this case, near the supply voltage level, as the amplifier is imbalanced. Then, the counter increases the input code for DAC. The VGA current-voltage conditions are adjusted accordingly to the DAC outputs, which are gradually changing in the opposite direction (until they swap their initial states 0 and the full scale). This process is continuously monitored by the control logic block, which terminates it immediately after $V_{\text {OUT-OFF }}$ reaches the lowest possible value. After that, the DAC continually supplies the last set analog outputs, and the calibrated VGA can emerge its normal operation.

The control logic is composed of two comparators with hysteresis. The VGA outputs are connected to one of the comparators inputs. The second input of both comparators is fed with the reference voltage $V_{\mathrm{REF}}$, which represents the ideal VGA output common mode. As explained previously, the DACs outputs starts to change as the counter begins to increase its output code. One of them is stepping down from the full scale and the other is stepping up from 0. Initially, both comparators are in low state at the output. The following NOR gate output is kept in high state. In this way, one of the subsequent NAND gate inputs is at high logic level, allowing

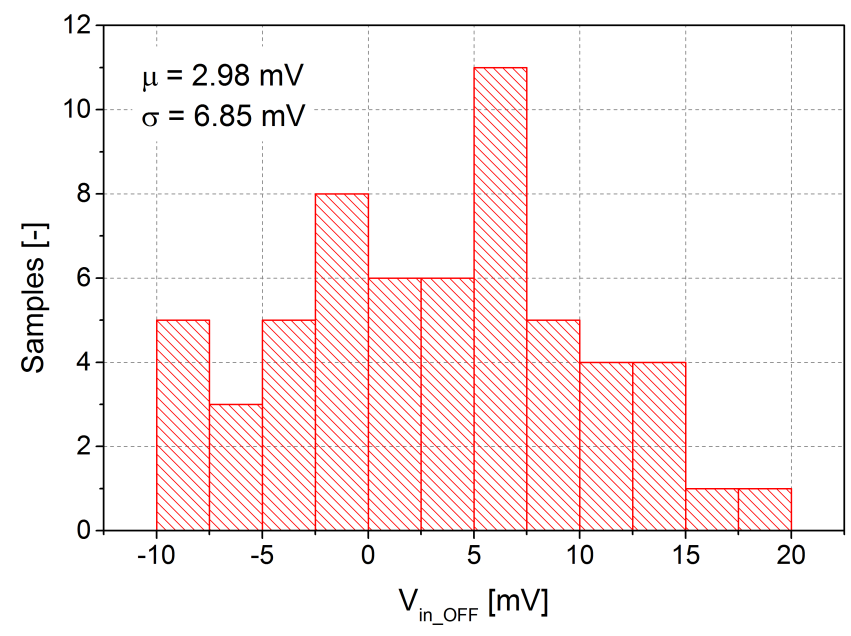

Fig. 20. The statistical distribution of $V_{\mathrm{IN}-\mathrm{OFF}}$ measurement over 60 samples.

the gate to be transparent for the other input. This is formed by the clock signal (CLK), which is then fed to the counter. In this manner, the DAC conversion proceeds and $V_{\mathrm{IN}-\mathrm{OFF}}$ is reduced.

After one of VGA outputs exceeds the $V_{\mathrm{REF}}$ value, the corresponding comparator flips its output to high level, and the NOR gate flips its output to low level. In this way, the CLK signal for compensation circuitry is stopped and the counter and DAC maintain their actual output values. If the calibration is terminated, control logic continues to sense the VGA output and it starts the whole cycle again when $V_{\text {IN-OFF }}$ exceed the given value. Therefore, this method is able to eliminate also the temperature and ageing drifts of the offset.

\subsection{Charge Pump for Self-powered Systems}

As reviewed and summarized exhaustively in rather recent publication [43], ULV CMOS based charged pumps (CP) are widely used within energy-autonomous or also called self-powered electronic systems. The most dominant advantage of CPs - fully on-chip integration capability - can be very challenging in terms of finding compromise between performance and acceptable area consumption, especially in ULV applications. Thus, this subsection presents the design of a dynamic threshold charge pump using a novel driver developed for ULV applications.

Our research was focused on pushing the minimum start-up voltage to its lower possible limit by improving the driving capability of the inverter-based driver and designing a boosting circuit for the $\mathrm{CP}$ cell itself as the future step. In start-up process, the driver is usually fed by the output voltage drawn from an energy harvester. Therefore, its driving capability strongly depends on it due to higher switch-on $R C$ constant. Also lowering $R C$ time constant results in higher switching frequency opportunity in ultra-low supply voltage conditions. Consequently, the enhancement of $V_{\mathrm{GS}}$ enables the use of much smaller transistors, then strongly reduces transistor capacitances and also switching losses. 


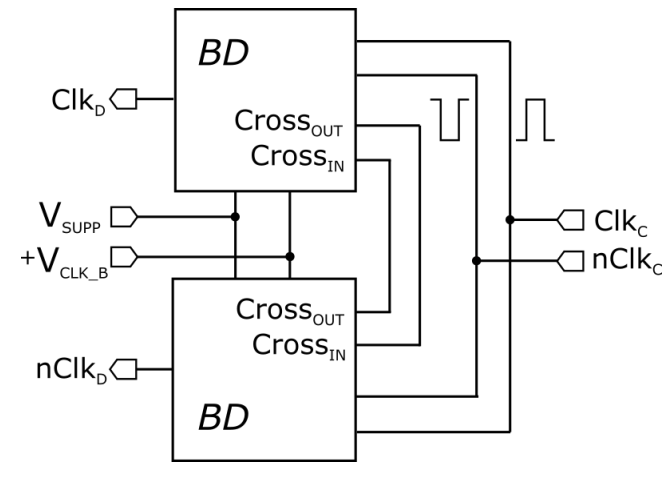

Fig. 21. Block diagram of the cross-connected driver [45].

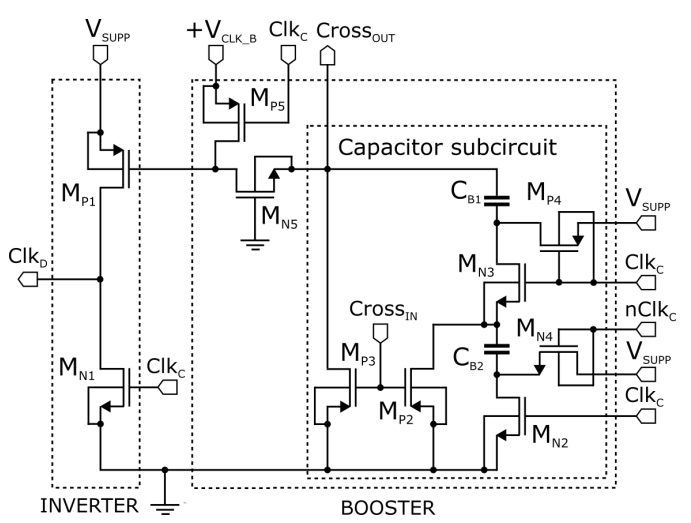

Fig. 22. Transistor level schematic of the proposed driver [45]

To improve the reliable start-up and operation conditions of the charge pump, the novel boosted driver for pull-up (PMOS) transistor has been proposed (Fig.21 and 22). The initial goal was to ensure the reliable function of the $\mathrm{CP}$ from the minimum possible value of the supply voltage. The boosting technique extends the range of driving voltage of $M_{P 1}$ transistor to $\approx-2 V_{\text {SUPP }} \div V_{\text {SUPP }}$ and reduces its ON resistance. The driver generates a non-overlapping outputs under the condition of non-overlapping control signals $\left(C l k_{\mathrm{c}}\right.$ and $n C l k_{\mathrm{c}}$ ). Design utilizes only two capacitors and charging is accomplished directly by the supply voltage, compared to three capacitors (two capacitors version is also available) with lower driving range of $\approx-V_{\text {SUPP }} \div V_{\text {SUPP }}$ and charging supported by the clock signal presented in [46]. More details together with the propagation delay measurement of the proposed driver can be found in [45] and [47] respectively.

To verify the performance of the proposed driver in ULV systems, the driver has been implemented as part of a CPbased energy harvesting control loop, as shown in Fig.23. Conventional On/Off regulation scheme has been chosen to maximize the charge pump efficiency. This scheme is based on a low-power BD rail-to-rail comparator [39] that blocks or passes clock signal to the driver. The switching frequency $f_{\text {CLK }}$ and size of the bootstrap capacitors and switching transistors have been designed as a trade-off between the reliable function of the driver from $200 \mathrm{mV}$ supply voltage in all corners and the temperatures range from $-20^{\circ} \mathrm{C}$ to $85^{\circ} \mathrm{C}$. Taking this into account, the bootstrap capacitors of $\approx 4 \times 15 \mathrm{pF}$ have been chosen with the expected charging capability up to $1 \mathrm{MHz}$.

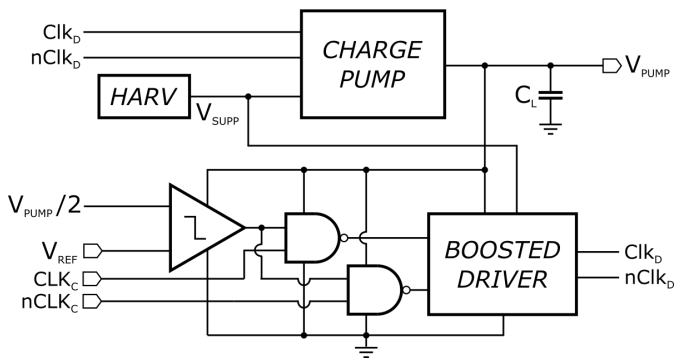

Fig. 23. The block diagram of the proposed charge pump system [45].

The charge pump consist of a three-stage dynamic threshold cross-coupled CP structure, where the number of stages was limited by available chip area, which limits the voltage conversion to $2-3 \times$ [44]. This restriction can be however, avoided by cascading a multiple chip due to identical topology of the individual CP cells. The crosscopupled CP belongs to popular, dual-branch linear types of CPs, having several advantages: $a$ ) good compromise between the power consumption and voltage conversion efficiency, $b$ ) compensated dual-branch topology reduces the output ripples and improves the pumping efficiency due to a flying capacitor size reduction resulting in faster charge transferring, $c$ ) reduction of loss between the last stage and the output capacitor since one of the two branches always provides current to keep the output voltage stable, $d$ ) lower reverse charge sharing, $e$ ) two phase non-overlapping clock can ensure sufficient timing of the switching process, $f$ ) the channel charge injection is eliminated due to complementary switching of adjacent NMOS/PMOS transistors. In spite of the above mentioned advantages, the application of the original cross-coupled topology is not suitable for ULV systems due to missing bootstrap techniques, and dynamic threshold method itself seems to be insufficient (see comparison to the other solutions in Tab. 4). In addition, the cross-coupled CP suffers from the reduced $V_{\mathrm{GS}}$ in the last stage, which is even more pronounced in regulated systems.

The proposed CP system was fabricated in a triple-well UMC $130 \mathrm{~nm}$ CMOS technology, where the CP core, control logic and the driver occupy area of $\approx 0.122 \mathrm{~mm}^{2}$ (Fig. 24). Early characterization process of the fabricated chip was primary aimed at investigating the reliable start and achieved parameters has been compared to other works [47]. The measurements proven that the control unit and the driver can work in the case of hot-start from the minimum supply voltage of $126 \mathrm{mV}$ at $160 \mathrm{kHz}$. Regulated voltage of $379 \mathrm{mV}$ was achieved where a regulatory deviation was caused by comparable impedances of electrostatic discharge (ESD) diodes with resistor based voltage divider. For more details of measurement setup please refers the [47]. The further measurements will be dedicated to characterization of the proposed $\mathrm{CP}$ systems in terms of others important parameters such as the maximum load capabilities, voltage conversion and power efficiency, etc. Table 4 summarizes the achieved parameters and compares the developed CP to other works. 


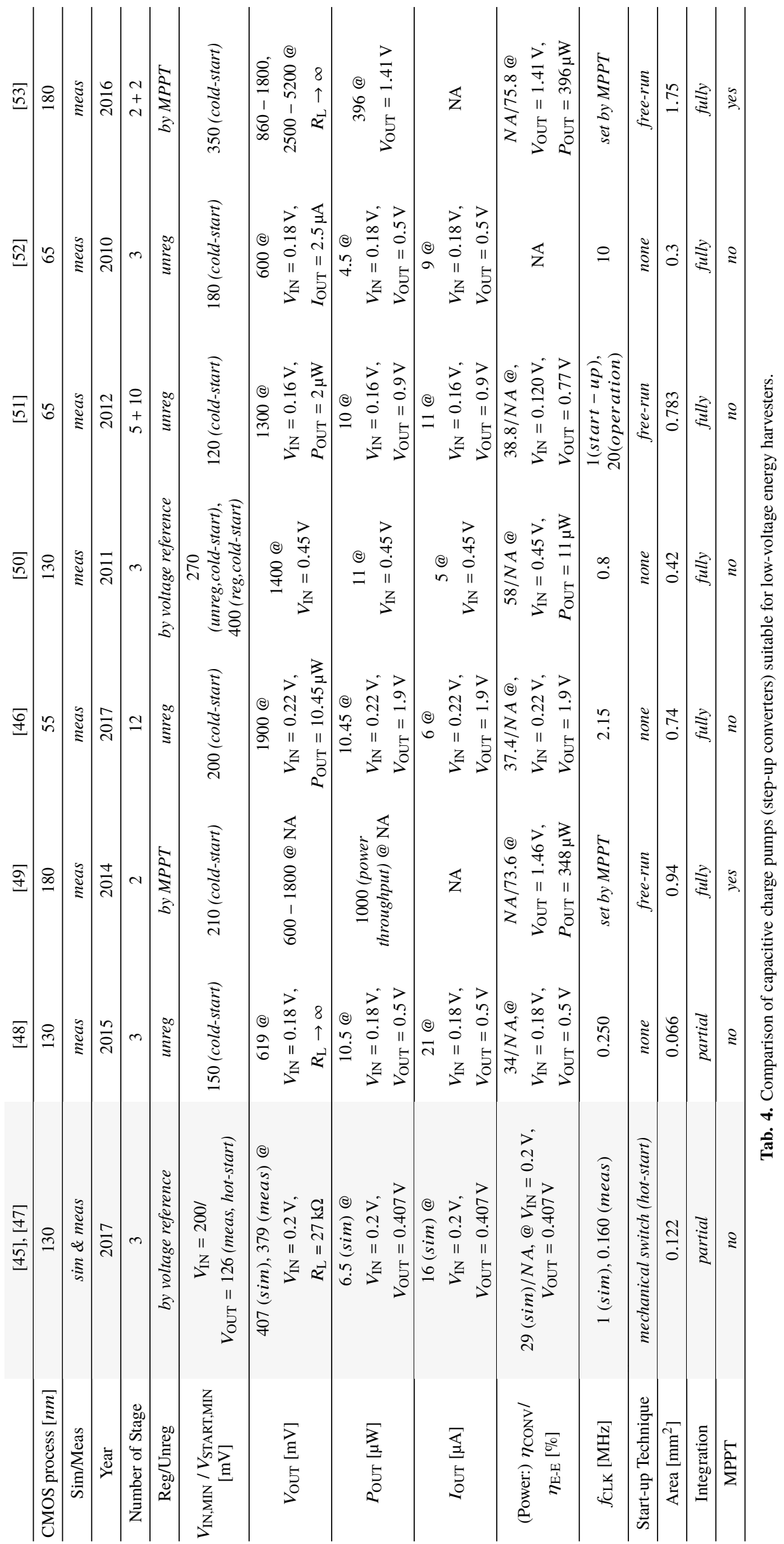




\subsection{Experimental Chip}

Figure 24 shows a microphotography of the developed experimental chip where the presented ULV analog circuit examples are implemented. The experimental chip was designed and implemented in a standard $130 \mathrm{~nm}$ CMOS technology and occupies area with dimensions of $1.2 \mathrm{~mm} \times 1.2 \mathrm{~mm}\left(1.44 \mathrm{~mm}^{2}\right)$. The charge pump together with the low-voltage driver together and the comparator are used as self-powered system. We have to note that chip do not contains the integrated clock generator yet and clock signals with desired frequency as well as the reference voltage for the comparator were generated externally. Both variablegain amplifiers are placed on the bottom of the chip. The BD current mirrors are located in the middle of the chip. The proper layout principle and techniques were employed in order to achieve good matching of all bulk-driven transistors used in the proposed analog circuits.

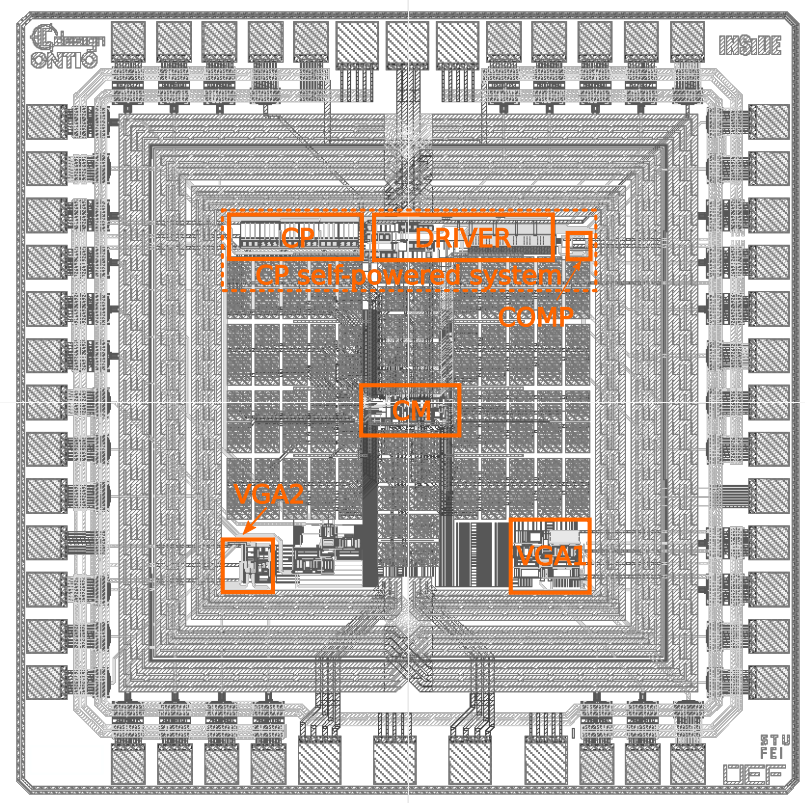

Fig. 24. Microphotography of the fabricated experimental chip.

\section{Conclusion}

Challenges and problems that have to be solved within the design of ultra low-voltage analog ICs are addressed in this paper. Since the use of standard circuit topologies as well as conventional design techniques is rather limited under the ultra low-voltage conditions, design engineers have to look for new topologies that are robust to PVT variations. As shown in this paper, the bulk-driven design technique can be an effective alternative for ULV analog ICs. Using this technique, the power consumption of analog circuits can be significantly reduced. However, the bulk driven approach can be used properly only for low-frequency applications. Therefore, the development of dedicated design techniques and analog topologies for ULV applications are still needed.

\section{Acknowledgements}

This work was supported by the Ministry of Education, Science, Research and Sport of the Slovak Republic under grant VEGA 1/0905/17 and the Slovak Research and Development Agency under grant APVV-15-0254.

\section{References}

[1] COURTLAND, R. Intel now packs 100 million transistors in each square millimeter (article). [Online] Cited 2008-06-23. Available at: https://spectrum.ieee.org/nanoclast/semiconductors/processors/intelnow-packs-100-million-transistors-in-each-square-millimeter

[2] SIU, S.-L., TAM, W.-S., WONG, H., et al. Influence of multifinger layout on the subthreshold behavior of nanometer MOS transistors. Elsevier Microelectronics Reliability, 2011, p. 1606-1609. DOI: 10.1016/j.microrel.2011.09.011

[3] BINKLEY, D. M. Tradeoffs and Optimization in Analog CMOS Design. Wiley, 2008. ISBN: 978-0-470-03136-0

[4] TSIVIDIS, Y. Operation and Modeling of the MOS Transistor. 2nd ed., Boston (MA): WCB/McGraw-Hill, 1999. ISBN: 9780070655232

[5] ENZ, CH., VITTOZ, E. A. Charge-based MOS Transistor Modeling: The EKV Model for Low-power and RF IC Design. Hoboken (NJ): John Wiley, 2006. ISBN: 9780470855416

[6] RAZAVI, B. Design of Analog CMOS Integrated Circuits. 1st ed., New York, NY (USA): McGraw Hill, 2001. ISBN: 0-07-238032-2

[7] ROY, K., MUKHOPADHYAY, S., MAHMOODI-MEIMAND, $\mathrm{H}$. Leakage current mechanisms and leakage reduction techniques in deep-submicrometer CMOS circuits. Proceedings of the IEEE, Feb 2003, vol. 91, no. 2, p. 305-327. DOI: $10.1109 /$ JPROC.2002.808156

[8] ZHAO, W., CAO, Y. New generation of predictive technology model for sub-45 $\mathrm{nm}$ early design exploration. IEEE Transactions on Electron Devices, Nov. 2006, vol. 53, no. 11, p. 2816-2823. DOI: 10.1109/TED.2006.884077

[9] BUCHER, M., LALLEMENT C., ENTZ, C., et al. Accurate MOS modelling for analog circuit simulation using the EKV model. In Proceedings of the IEEE International Symposium on Circuits and Systems. Circuits and Systems Connecting the World. (ISCAS 96). Atlanta, GA (USA), 1996, p. 703-706, vol. 4. DOI: 10.1109/ISCAS.1996.542121

[10] ONABAJO, M., SILVA-MARTINEZ, J. Analog Circuit Design for Process Variation-resilient Systems-on-a-chip. Springer Science \& Business Media, 2012.

[11] CHANG, J., CHEN, Y., CHAN, W., et. al. A $7 \mathrm{~nm} 256 \mathrm{Mb}$ SRAM in high-k metal-gate finFET technology with write-assist circuitry for low-VMIN applications. In Proceedings of the IEEE International Solid-State Circuits Conference. 2017, p. 206-208. DOI: $10.1109 /$ ISSCC.2017.7870333

[12] SCHRODER, D. K. Negative bias temperature instability: What do we understand? In Proceedings of the Microelectronics Reliability. 2007, p. 841-852. ISSN: 0026-2714

[13] KUMAR, R., KURSUN, V. Voltage optimization for temperature variation insensitive CMOS circuits. In Proceedings of the 48th Midwest Symposium on Circuits and Systems. Covington, KY, 2005, p. 476-479. DOI: 10.1109/MWSCAS.2005.1594141

[14] WOLPERT, D., AMPADU, P. Managing Temperature Effects in Nanoscale Adaptive Systems. Springer New York, 2012. ISBN: 978-1-4614-0748-5 
[15] RODRIGUEZ-VILLEGAS, E., BARNES, H. Solution to trapped charge in FGMOS transistors. Electronics Letters, 2003, vo. 39, no. 19 , p. 1416-1417. DOI: 10.1049/el:20030900

[16] BAEK, K. J., GIM, J. M., KIM, H. S., et al. Analogue circuit design methodology using self-cascode structures. Electronics Letters, 2013, vol. 49, no. 9, p. 591-592. DOI: 10.1049/el.2013.0554

[17] DUQUE-CARILlO, J. F., AUSIN, J. L., TORELLI, G., et al. 1$\mathrm{V}$ rail-to-rail operational amplifiers in standard CMOS technology. IEEE Journal of Solid-State Circuits, 2000, vol. 35, no. 1, p. 33-44. DOI: $10.1109 / 4.818918$

[18] KUMAR, M., SANDEEP, A., SUJATA, P. Level shifter design for low power applications. International Journal of Computer Science and Information Technology (IJCSIT), 2010, vol. 2, no. 5, p. 124-132. DOI: $10.5121 /$ ijcsit.2010.2509

[19] WANG, A., CAlhoun, B. H., Chandrakasan, A. P. Sub-Threshold Design for Ultra Low-Power Systems. 1st ed., New York (USA): Springer Science+Business Media, 2006. ISBN: 0-387-33515-3

[20] SHOULI, Y.,SANCHEZ-SINENCIO, E. Low voltage analog circuit design techniques: A tutorial. In Proceedings of the IEICE Transactions on Fundamentals of Electronics, Communications and Computer Sciences, 2000, vol. 83, no. 2, p. 179-196. ISSN: 0916-8508

[21] CUNHA, A. I. A, SCHNEIDER, M. C., GALUP-MONTORO, C. An MOS transistor model for analog circuit design. IEEE Journal of Solid-State Circuits, 1998, vol. 33, no. 10, p. 1510-1519. DOI: $10.1109 / 4.720397$

[22] BLALOCK, B. J., ALLEN, P. E., RINCON-MORA, G. A. A 1 v CMOS op amp using bulk-driven MOSFETs. In Proceedings of the IEEE International Solid-State Circuits Conference (ISSCC). San Francisco, CA (USA), 1995, p. 192-193. DOI: $10.1109 /$ ISSCC. 1995.535518

[23] TSIVIDIS, Y. Mixed Analog-Digital VLSI Devices and Technology. 2nd ed., Singapore: World Scientific Publishing Co., 2002. ISBN: 978-981-238-111-8

[24] BLALOCK, B. J., ALLEN, P. E. A low-voltage, bulk-driven MOSFET current mirror for CMOS technology. In Proceedings of the IEEE International Symposium on Circuits and Systems (ISCAS '95). Seattle, WA (USA), 1995, p. 1972-1975, vol. 3. DOI: 10.1109/ISCAS.1995.523807

[25] ARBET, D., STOPJAKOVA, V., KOVAC, et al. $130 \mathrm{~nm}$ CMOS bulkdriven variable gain amplifier for low-voltage applications. Journal of Circuits, Systems and Computers, 2017, vol. 26, no. 8, p. 1-19. DOI: $10.1142 / \mathrm{S} 0218126617400035$

[26] NIRANJAN, V., KUMAR, A., JAIN, S. B. Low voltage flipped voltage follower based current mirror using DTMOS technique. In Proceedings of the International Conference on Multimedia, Signal Processing and Communication Technologies (IMPACT-2013). Aligarh (India), 2013, p. 250-254. DOI: 10.1109/MSPCT.2013.6782129

[27] ZUO, L. Low-Voltage Bulk-Driven Amplifier Design and Its Application in Implantable Biomedical Sensor. (Ph.D. thesis). University of Tennessee, Knoxville (USA), 2012.

[28] XANTHOPOULOS, C., et al. IC laser trimming speed-up through wafer-level spatial correlation modeling. In Proceedings of the 2014 International Test Conference. 2014, p. 1-7. DOI: 10.1109/TEST.2014.7035329

[29] GODOY, P., DAWSON, J. L. Chopper stabilization of analog multipliers, variable gain amplifiers, and mixers. IEEE Journal of Solid-State Circuits, 2018, vol. 43., no. 10, p. 2311-2321. DOI: $10.1109 /$ JSSC.2008.2004328
[30] DANCHIV, A., BODEA, M. An area efficient low offset autozero amplifier design. In Proceedings of the Ph.D. Research in Microelectronics and Electronics. 2009, p. 140-143. DOI: 10.1109/RME.2009.5201331

[31] PASTRE, M., KAYAL, M. Methodology for the Digital Calibration of Analog Circuits and Systems. 1st ed., Springer, 2006. ISBN: 978-1-4020-4253-9

[32] RAKUS, M., STOPJAKOVA, V., ARBET, D. Analysis of bulk-driven technique for low-voltage IC design in $130 \mathrm{~nm}$ CMOS technology. In Proceedings of the 15th International Conference on Emerging eLearning Technologies and Applications (ICETA). 2017, p. 1-6. DOI: 10.1109/ICETA.2017.8102522

[33] ElZAKKer, M., TUIJL, E., GERAEDTS, P., et al. A 10-bit charge-redistribution ADC consuming $1.9 \mu \mathrm{W}$ at $1 \mathrm{MS} / \mathrm{s}$. IEEE Journal of Solid-State Circuits, 2010, vol. 45, no. 5, p. 1007-1015. DOI: $10.1109 /$ JSSC.2010.2043893

[34] MIYAHARA, M., ASADA, Y., PAIK, D.,et al. A low-noise self-calibrating dynamic comparator for high-speed ADCs. IEEE Asian Solid-State Circuits, 2008, vol. 56, no. 8, p. 269-272. DOI: 10.1109/ASSCC.2008.4708780

[35] SARKAR, S., BANERJEE, S. $500 \mathrm{MHz}$ differential latched current comparator for calibration of current steering DAC. IEEE Students' Technology Symposium (TechSym). 2014, p. 309-3012. DOI: 10.1109/TechSym.2014.6808066

[36] FAN, S., WEI, R., ZHAO, L., et al. An ultra-low quiescent current power management system with maximum power point tracking (MPPT) for battery-less wireless sensor applications. IEEE Transactions on Power Electronics, 2013, no. 99, p. 1-1. DOI: 10.1109/TPEL.2017.2769708

[37] AKBARI, M., MAYMANDI-NEJAD M., MIRBOZORGI, S. A. A new rail-to-rail ultra low voltage high speed comparator. In Proceedings of the 21st Iranian Conference on Electrical Engineering. 2013, p. 1-6.

[38] LI, Y., MAO, W., ZHANG, Z., et al. An ultra-low voltage comparator with improved comparison time and reduced offset voltage. In Proceedings of the IEEE Asia Pacific Conference on Circuits and Systems. 2014, p. 407-410. DOI: 10.1109/APCCAS.2014.7032806

[39] NAGY, L., ARBET, D., KOVAC, M., et al. Low-power bulk-driven rail-to-rail comparator in $130 \mathrm{~nm}$ CMOS technology. In Proceedings of the IEEE AFRICON 2017. Cape Town (South Africa), 2017, p. 649-652. DOI: 10.1109/AFRCON.2017.8095559

[40] ARBET, D., KOVAC, M., NAGY, L., et al. Low-voltage bulk-driven variable gain amplifier in $130 \mathrm{~nm}$ CMOS technology In Proceedings of the 2016 IEEE 19th International Symposium on Design and Diagnostics of Electronic Circuits Systems (DDECS). Kosice (Slovakia), 2016, p. 1-6. DOI: 10.1109/DDECS.2016.7482439

[41] ARBET, D., KOVAC, M., NAGY, L., et al. Variable-gain amplifier for ultra-low voltage applications in $130 \mathrm{~nm}$ CMOS technology. In Proceedings of the 2016 39th International Convention on Information and Communication Technology, Electronics and Microelectronics (MIPRO). Opatija, 2016, p. 51-56.

[42] RAIKOS, G., VLASSIS, S. $0.8 \mathrm{~V}$ bulk-driven variable gain amplifier. In Proceedings of the 2010 17th IEEE International Conference on Electronics, Circuits, and Systems (ICECS). 2010, p. 347-350. DOI: $10.1109 /$ ICECS.2010.5724524

[43] MI, M. L. S., ISLAM, M. S., SAMPE, J., et al. Review of charge pump topologies for micro energy harvesting systems. American Journal of Applied Sciences, 2016, vol. 13, no. 5, p. 628-645. DOI: 10.3844/ajassp.2016.628.645

[44] NAGY, G., ARBET, D., STOPJAKOVA, V., et al. Novel CMOS bulkdriven charge pump for ultra low input voltage. Radioengineering, 2016, vol. 25 , no. 2 , p. 321-331. DOI: 10.13164/re.2016.0321 
[45] SOVCIK, M., KOVAC, M., ARBET, D., et al. Ultra-low-voltage driver for large load capacitance in $130 \mathrm{~nm}$ CMOS technology. In Proceedings of the IEEE 20th International Symposium on Design and Diagnostics of Electronic Circuits Systems (DDECS), 2017, p. 127-132. DOI: 10.1109/DDECS.2017.7934567

[46] INTASCHI, L., BRUSCHI, P., IANNACCONE, G., et al. A 220-mV input, 8.6 step-up voltage conversion ratio, 10.45 - $\mu \mathrm{W}$ output power, fully integrated switched-capacitor converter for energy harvesting. In Proceedings of the IEEE Custom Integrated Circuits Conference (CICC). 2017, p. 1-4. DOI: 10.1109/CICC.2017.7993623

[47] SOVCIK, M., KOVAC, M., ARBET, D., et al. Ultra-low-voltage boosted driver for self-powered systems. Microelectronics Reliability, 2018, vol. 80, p. 155-163. DOI: 10.1016/j.microrel.2017.11.006

[48] KIM, J., MOK, P. K. T., KIM, C. A 0.15 V input energy harvesting charge pump with dynamic body biasing and adaptive dead-time for efficiency improvement. IEEE Journal of Solid-State Circuits, 2015, vol. 50, no. 2, p. 414-425. DOI: 0.1109/JSSC.2014.2375824

[49] OZAKI, T., HIROSE, T., NAGAI, T., et al. A 0.21-V minimum input, $73.6 \%$ maximum efficiency, fully integrated voltage boost converter with MPPT for low-voltage energy harvesters. In Proceedings of the 40th European Solid State Circuits Conference (ESSCIRC). 2014, p. 255-258. DOI: 10.1109/ESSCIRC.2014.6942070

[50] SHIH, Y. C., OTIS, B. P. An inductorless DC-DC converter for energy harvesting with a $1.2-\mu \mathrm{W}$ bandgap-referenced output controller IEEE Transactions on Circuits and Systems II: Express Briefs, 2011, vol. 58, no. 12, p. 832-836. DOI: 10.1109/TCSII.2011.2173967

[51] CHEN, P. H., ISHIDA, K., ZHANG, X., et al. 120-mV input, fully integrated dual-mode charge pump in 65-nm CMOS for thermoelectric energy harvester. In Procedings of the 17th Asia and South Pacific Design Automation Conference. 2012, p. 469-470. DOI: 10.1109/ASPDAC.2012.6164994

[52] CHEN, P. H., ISHIDA, K., ZHANG, X., et al. 0.18-V input charge pump with forward body biasing in startup circuit using $65 \mathrm{~nm}$ CMOS. IEEE Custom Integrated Circuits Conference 2010, 2010, p. 1-4. DOI: 10.1109/CICC.2010.5617444

[53] OZAKI, T., HIROSE, T., ASANO, H., et al. Fully-integrated high-conversion-ratio dual-output voltage boost converter with MPPT for low-voltage energy harvesting. IEEE Journal of Solid-State Circuits, 2016, vol. 51, no. 10, p. 2398-2407. DOI: $10.1109 /$ JSSC.2016.2582857

[54] MIN, K. S., KIM, Y. H., AHN, J. H., et al. CMOS charge pumps using cross-coupled charge transfer switches with improved voltage pumping gain and low gate-oxide stress for low-voltage memory circuits. In IEEE International Symposium on Circuits and Systems. 2002, vol. 5, p. V-V. DOI: 10.1109/ISCAS.2002.1010761

[55] KI, W. H., LU, Y., SU, F., et al. Analysis and design strategy of on-chip charge pumps for micro-power energy harvesting applications. In Proceedings of the IFIP/IEEE International Conference on Very Large Scale Integration-System on a Chip. 2011, p. 158-186. DOI: 10.1007/978-3-642-32770-4_10

\section{About the Authors ...}

Viera STOPJAKOVÁ received the M.Sc. and the Ph.D. degrees in Microelectronics from Slovak University of Technology in Bratislava, Slovakia in 1992 and 1997, respectively.
Currently, she is a full-time Professor at the Institute of Electronics and Photonics of the same university. She has been involved in several EU funded research projects under different funding schemes and also in national research grants. She has published over 180 papers in various journals and conference proceedings; and she is a co-inventor of two US patents in the field of on-chip supply current testing. Her main research interests include IC design and test, on-chip testing, low-voltage analog IC design, smart sensors, on-chip energy harvesting and biomedical monitoring.

Matej RAKÚS received the M. S. degree in Electronics from Slovak University of Technology in Bratislava in 2015. Since September 2015 he has been a PhD student at the institute of Electronics and Photonics of the same university. His main research interests are low-voltage and low-power IC design with focus to techniques using bulk-driven MOS transistors and MOS transistors operating in sub-threshold region.

Martin KOVÁČ received his M.S. degree in Electronics from Slovak University of Technology in Bratislava, Slovakia in 2013. He is currently a $\mathrm{Ph}$. D. student under the supervision of Prof. Viera Stopjakova. His research is focused on low-voltage low-power analog IC and UWB antenna design, on-chip parametric testing, energy-efficient RF integrated systems for biomedical applications.

Daniel ARBET received the M.S. degree and Ph.D. degree in Electronics from Slovak University of Technology in Bratislava, Slovakia in 2009 and 3013, respectively. Currently, he is a researcher at the Institute of Electronics and Photonics. He has published more than 40 papers. His main research interests are low-voltage low-power analog and mixed-signal IC design, on-chip parametric testing, analog BIST and test implementation.

Lukáš NAGY received his M.S. and PhD. degrees at Slovak University of Technology in 2008 and 2012, respectively. He currently works as a researcher at the Institute of Electronics and Photonics at the same university. His main research interests include the device modeling, analog and mixed-signal IC design, SoC design and asynchronous systems design.

Michal SOVČÍK received the M.S. degree in Electronics from Slovak University of Technology in Bratislava, Slovakia in 2016. Since September 2016 he has been a $\mathrm{PhD}$ student at the Institute of Electronics and Photonics of the same university. His main research interest is the low-power IC design aimed at digital calibration for analog ICs.

Miroslav POTOČNÝ received the M.S. degree in Electronics from Slovak University of Technology in Bratislava, Slovakia in 2016. Since September 2016 he has been a PhD student at the Institute of Electronics and Photonics of the same university. His main research interest is design of RF integrated circuits. 